Improving national cultural indices using a longitudinal meta-analysis of Hofstede's dimensions

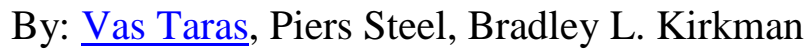

Taras, V., Steel, P., Kirkman, B. (2012). Improving National Cultural Indices Using a MetaAnalysis of Hofstede's Dimensions. Journal of World Business, 47(3): 329-341

Made available courtesy of Elsevier: https://doi.org/10.1016/j.jwb.2011.05.001

\begin{abstract}
(c) (i) (9) $९$ This work is licensed under a Creative Commons AttributionNonCommercial-NoDerivatives 4.0 International License.
\end{abstract}

\begin{abstract}
:
This meta-analysis offers an updated set of national cultural scores along the dimensions of Hofstede's cultural framework. The meta-analytic national cultural indices have two advantages. First, they are based on a larger and more representative sample than that used in Hofstede's or any other cross-cultural comparison study. The data come from 451 empirical studies representing over 2000 samples comprising over half a million individuals from 49 countries and regions. Second, cultural change is addressed by offering separate sets of indices for different decades. This would be helpful in longitudinal studies of the interplay between culture and other phenomena. The meta-analytic indices are validated against a set of external criteria.
\end{abstract}

Keywords: Culture | Cultural change | Meta-analysis | Hofstede

Article:

The slowest one now will later be fast As the present now will later be past.

The order is rapidly fadin'.

And the first one now will later be last

For the times they are a-changin'.

\title{
Bob Dylan
}

Over the last several decades, culture has been one of the key research constructs in fields ranging from management and psychology to accounting and marketing. This interest was largely triggered by Hofstede's work, detailed in his 1980 book entitled, Culture's Consequences. Although culture had been researched much earlier than Hofstede's work (e.g., Kuhn \& McPartland, 1954; Rokeach, 1973), he was the first to supplement a theoretical model with an elegant set of indices that quantitatively described national cultures. Consequently, Hofstede's work led to an explosion of cross-cultural research in business, psychology, and other disciplines that favor quantitative research methods. 
Hofstede's cultural scores provided a foundation for crosscultural corporate training and international management courses in business and executive education curricula. The indices not only aided generations of managers and practitioners working in international settings, but also had a profound impact on subsequent cross-cultural research (Taras \& Steel, 2009). According to Google Scholar,the total citation countfor various editions of Culture's Consequences is currently over twenty thousand. Hofstede's model of culture has been successfully applied in research on a wide range of organizational and national issues, including leadership, teamwork, justice, communication, ethics, satisfaction, commitment, foreign market entry modes, international trade, and individual, company and national performance, to name a very few (see Taras, Kirkman, \& Steel, 2010 for a meta-analytic review). Most importantly, his original cultural indices have been used in thousands of studies including a large number published in recent years (e.g., Chakrabarty, 2009; Newburry \& Yakova, 2006; Ng, Sorensen, \& Yim, 2009).

Despite their enormous popularity, Hofstede's cultural indices are not without limitations. First, Hofstede's research design raises questions about the generalizability of his findings (e.g., McSweeney, 2002; Voronov \& Singer, 2002). Although over 60,000 people participated in the survey, some countries were represented by less than a hundred respondents. Moreover, the study was based on a convenience sample of employees in a single American organizationIBM. As noted by Hofstede, a uni-organizational design can be advantageous as it minimizes the effects of external factors (for more details see Hofstede, 2002). However, this approach can also severely limit data generalizability to the broader population. This issue is particularly problematic for Hofstede as IBM has a strong organizational culture, and its employees tend to be from a distinct demographic profile. As noted by Schwartz (1994, p. 91), "highly educated well-paid IBM employees' ability to represent the general population likely differs from country to country, with the discrepancy probably being greater, for example, in the Third World nations ... than in industrialized Western nations" (p. 91).

Second, mounting evidence of cultural changes around the world (e.g., Inglehart \& Baker, 2000; Ralston, Holt, Terpstra, \& Kai Cheng, 1997) raises concerns whether or not Hofstede's indices derived from data collected in 1967-1973 are still relevant. It is uncertain if Hofstede's 40-year-old data can be reliably used in conjunction with variables representing contemporary phenomena or even with archival data from the 1990s as the relative rankings of national cultures may have changed in the past decades.

A number of scholars attempted to address the limitations of Hofstede's work by conducting new large-scale culture comparison studies (e.g., House, Hanges, Javidan, Dorfman, \& Gupta, 2004; Inglehart \& Baker, 2000; Schwartz, 1994; Smith, Dugan, \& Trompenaars, 1996). While the post-Hofstede research substantially advanced our understanding of culture, any subsequent study was, unfortunately, subject to the same limitations. That is, the lack of data generalizability across population and time. First, the high cost of collecting data internationally imposes restrictions on the representativeness and size of national samples. Managers, teachers, or students, convenient as they may be to sample, do not perfectly represent the larger society. Second, cultural indices offered by any single study necessarily represent a specific time period. This limits the ability to match the culture measures with data coming from other, earlier or later time periods. Even if we had the resources to re-administer the original cultural survey periodically over an extended time period, we still could not turn back the clock and administer the survey in days gone by. If we need to match older archival data with cultural indices for a corresponding period. 
A possible, heretofore unexplored, solution for addressing these limitations may be metaanalysis. While certainly far from perfect, the meta-analytic approach to national cultural rankings may be better suited for handling the issues of limited sample generalizability across populations and time periods. Around the world, researchers have conducted thousands of smaller studies based on Hofstede's model. This generated an array of compatible data that can be meta-analytically integrated into a dataset that would be the largest and most representative yet. Moreover, as the individual studies sampled the same populations (i.e., nations) at different times, meta-analytic data offers a longitudinal perspective. This opens up the possibility of examining cultures dynamically and exploring how and why cultures changes over time. By creating sets of cultural indices for each decade since Hofstede's work, as we do here, we allow for matching culture with other longitudinal datasets such as national economic development indicators to explore a host of new research questions pertaining to the causes and effects of cultural change. Furthermore, the dataset can not only allow us to look back in time, it can also be appended with data generated by future studies, further extending the timeframe. In this way, compared to conventional studies, a meta-analysis offers cultural indices that better generalize to their respective populations and account for, and reflect on, cultural changes.

We begin with an overview of Hofstede's cultural dimensions that are the foundation of our study, followed by a summary of issues and theories related to cultural change. Next, we describe how our meta-analytic data were collected, analyzed, and validated. Then, we report our meta-analytic decade-by-decade cultural indices and validate our findings against a set of theoretically relevant criteria. We conclude with a discussion of the implications of our findings and directions for future research.

\section{Overview of Hofstede's model of culture}

"Culture" is one of the most popular and widely used words. Google lists half a billion searches for "culture", more than for "politics", "money", or "sex." As with other pervasive words, defining culture is no easy task. Half a century ago, Kroeber and Kluckhohn (1952) found 164 distinct definitions of culture, and that number keeps growing (Taras, Rowney, \& Steel, 2009). Crosscultural researchers typically use Hofstede's (1980) definition of culture as " the collective programming of the mind which distinguishes the members of one human group from another' (p. 25). Although it is generally recognized that culture is a multilayered construct represented by values, practices and artifacts, as per the Hofstedian paradigm, cultural values have been the focus of most cross-cultural research (Taras et al., 2009; Taras \& Steel, 2009).

Hofstede's (1980) model of culture was initially comprised of four value dimensions: Power Distance - the extent to which the less powerful persons in a society accept inequality in power and consider it as normal; Individualism - the degree to which people prefer to act as individuals rather than as members of groups, in individualist cultures any person looks primarily after his/her own interest, while in collectivist cultures people are assumed to belong to tight ingroups that protect interest of its members in return for their loyalty; Masculinity - the degree to which masculine values like assertiveness, performance, success and competition prevail over feminine values like the quality of life, maintaining warm personal relationships, service, care for the weak, and solidarity; and Uncertainty Avoidance - the extent to which people are made nervous by situations which they perceive as unstructured, unclear, or unpredictable. Long- vs. Short-Term Orientation dimension (a.k.a. Confucian Dynamism) was later added to the model, but it has been rarely used and could not be included in our meta-analysis due to the lack of data. 


\section{Cultural change}

Traditionally, it has been assumed that culture is effectively unchangeable. Hofstede viewed theories of cultural change as "nai"ve" (Hofstede, 2001, p. 34) and stressed that cultural change would be extremely slow and should be measured in terms of generations and centuries, not decades. He predicted that relative national cultural scores would not change substantially "until at least 210000 (Hofstede, 2001, p. 36).

Nevertheless, there is growing empirical evidence, albeit scattered, showing that cultures are changing. This evidence suggests that change is certainly not rapid, but it appears to be occurring faster than expected by Hofstede. The World Value Survey that tracked attitudes and values in 81 societies over several years found evidence of "a massive cultural change" even within the relatively brief period covered by the data (Inglehart \& Baker, 2000, p. 19). A number of studies documented a substantial change in values in some societies following the events of 9/11 (e.g., Murphy, Gordon, \& Mullen, 2004; Olivas-Luján, Harzing, \& McCoy, 2004). Furthermore, extreme cultural stability arguments belie cohort effects. However, cultural differences across cohorts, such as "Baby Boomers" and "Generation X", are well-documented (e.g., Smith, 2000). Most telling is Hofstede's own work. A portion of his sample was assessed twice over a 4-year period and "from a comparison between the two survey rounds, it became clear that there had been a worldwide shifts on some questions" (Hofstede, 2001, p. 53).

While the evidence of some level of cultural change is abundant, the pattern of change remains uncertain. It is not clear whether or not cultures change uniformly in different countries. It is likely that the change is faster in some regions than in others, which means that countries' relative positions in the national rankings are changing over time. Just because the U.S. was the most individualistic culture in the 1970s does not necessarily mean that it is still today.

Since our meta-analytic cultural indices are based on a larger and more representative dataset than that used in Hofstede's or any other culture comparison study, this should extend the useful life of Hofstede's framework by mitigating the effect of any observed cultural change. By providing separate sets of indices for each of the past several decades, researchers can match archival data with culture data from corresponding time periods. If cultural change is indeed happening in a matter of decades, as suggested by the World Value Survey (Inglehart \& Baker, 2000) and other cultural dynamics studies (e.g., Olivas-Luja'n et al., 2004; Shah, 2009), then using older indices may pose a serious threat to validity. For example, a researcher may be exploring the effects that culture has on a particular phenomenon, as measured in an archival dataset from 1990. Pairing these data with Hofstede's cultural indices from 1967 to 1973 may provide misleading results as there would be a gap of 20 years between the measures of culture and the archival dataset. Similarly, there would also be a gap of 20 years if a researcher collected culture data today to match it with the data from the 1990s. The decade-by-decade metaanalytic scores provided in the present study allow for a closer temporal match between cultural data and archival or newly collected data.

In addition, the meta-analytic indices permit a longitudinal perspective into studies of culture, as well as explore not only the consequences of culture, where cultural shifts bring about later societal changes, but also explore culture as consequence, where societal changes brings about cultural shifts (Steel \& Taras, 2010). The following section details our data collection, coding, analysis, and validation procedures.

\section{Method}




\subsection{Inclusion criteria}

Our meta-analytic database includes studies that reported cultural values of their participants measured using models and methodology comparable with those devised by Hofstede. A common challenge in meta-analysis is the tradeoff between sample size and commensurability. No two studies are identical but differ in terms of their methodology, metric, or sample. Minor instrument modifications, such as changes in the scale range (e.g., 1-5 or 1-7) or sequence of survey items, are not likely to significantly alter the construct. However, if the studies are substantively different, integration becomes questionable due to commensurability or the "apples and oranges" problem (Sharpe, 1997). The commensurability concerns push for stricter study inclusion criteria. Relaxing inclusion standards allows for more studies to be included in the meta-analytic dataset. Larger sample size not only increases the generalizability and reliability of the data, but also allows the assessment of more target populations across a wider range of conditions and at more precise degrees of time.

To ensure the optimal balance between sample size and method variance, we took a content validation approach in which multiple coders determine instrument compatibility by conducting a thorough comparative item analysis. Such use of expert judgment is typical of meta-analysis in general as well as of previous metaanalyses that specifically focused on Hofstede's framework (Oyserman, Coon, \& Kemmelmeier, 2002; Steel \& Taras, 2010; Taras, Kirkman, \& Steel, 2010). Using information from Taras' (2009) Catalogue of Instruments for Measuring Culture, we examined construct definitions and individual items of 135 culture measurement instruments for their consistency with construct definitions originally introduced by Hofstede and the items in the VSM-82 and VSM-94 versions of his instrument. We kept our assessment purposefully conservative. When in doubt we excluded the measure, preferring to make errors of omission rather than commission. After several rounds of inspection, depending on the dimension, only 8-26 (roughly 1 in 10) of the considered instruments qualified for inclusion. For example, a number of instruments that were included in the widely cited Oyserman et al.'s (2002) meta-analysis of Individualism-Collectivism, such as those developed by Wagner and Moch (1986), did not satisfy our stricter selection criteria. As a result, data collected using different versions of the Hofstede's original VSM accounted for $77.0 \%$ of all Power Distance observations, 66.4\% of Masculinity, 64.5\% of Uncertainty Avoidance, and $31.6 \%$ of Individualism. The lower percentage for Individualism reflects the fact that it has been the most popular dimension and a great number of alternative instruments have been developed to measure the construct.

Our inclusion decisions were further supported by a number of indicators. First, most of the instruments included in our pool were explicitly based on Hofstede's definitions of cultural constructs and items from his VSM (e.g., Dorfman \& Howell, 1988; Furrer, Liu, \& Sudharshan, 2000; Vitell, Paolillo, \& Thomas, 2003). Second, a number of studies provided empirical support for convergent validity between Hofstede's VSM and other instruments subsequently developed to measure the same constructs. For example, Schimmack, Oishi, and Diener (2005) showed that Individualism measures developed Singelis, Triandis, Bhawuk, and Gelfand (1995) and Triandis and Gelfand (1998), the two most popular instruments in our meta-analytic dataset, converge with data obtained using the VSM. Similarly, Maznevski, DiStefano, Gomez, Noorderhaven, and $\mathrm{Wu}(2002)$ state that their measures produce scores "consistent with his (Hofstede's) results" (p. 287). Finally, the strongest argument in support of convergence of our VSM and non-VSM subsets are the correlations between the two sets of indices. They were 0.66 for Power Distance, 
0.75 for Individualism, 0.60 for Masculinity and 0.65 for Uncertainty Avoidance, as high as the test-retest reliability ofthe VSM, which is $0.61-0.75$ depending on the dimension and sample. In other words, VSM subset correlates as well with the scales included in our analysis as it does with itself. The correlations would have likely been even higher if the measures were administered identically and with the same respondents, as is typically done with test-retest reliability assessment. Consequently, it is difficult to conceive of better results that indicate equivalence.

\subsection{Literature search}

All available sources were searched for data comparable with those from Hofstede's IBM study. First, we conducted a computer search through all major electronic databases. Second, we conducted searches in almost 100 relevant journals for the 1980-2010 period (or since the journal was launched). Third, we reviewed the reference sections of each article being coded for links to publications potentially containing data for the meta-analysis. Fourth, we used Google Scholar's "cited by" function with every coded paper to check for links to relevant subsequent studies. Fifth, we sent requests for links to more studies through mailing list-serves of the Academy of International Business and the International Management Division of the Academy of Management. Sixth, we contacted all authors with more than three papers in our database with information about our meta-analysis and requested for links to additional published or unpublished studies. Of the 37 researchers in the pool, 29 provided additional references. Finally, we are especially thankful to Professor Hofstede who has been keeping track of the studies that have utilized his VSM and graciously shared his personal records with us.

\subsection{Coding and common metric}

The key information extracted from studies in our meta-analytic database included the sample means along the four cultural dimensions, sample size, year when and the country where the survey was conducted, as well as sample demographics. As in the IBM study, we grouped

underrepresented countries into cultural regions following Hofstede's original clustering scheme with some adjustments to account for changes in geopolitics of the 1990s. The following regions were formed: Africa (South Africa was analyzed separately), Arab countries, Caribbean, Central America, South America, Asian Republics of former USSR, Baltic Republics of former USSR, and Slavic Republics of former USSR.

To provide a foundation for the analysis, we converted all culture data into a common metric. First, the differences in the scale ranges (e.g., 1-5, 1-7) were resolved by transforming raw scores to the $0-1$ range. Then, we addressed the differences in item functioning and scoring schemes of different instruments by standardizing the scores (mean $=0, \mathrm{SD}=1)$ within data subsets corresponding to each instrument type in our pool. Consequently, our scores usually do not exceed 2 and +2 on the extremes with zero indicating a neutral position along a cultural dimension (corresponds to roughly 50 on Hofstede's original scale).

Another challenge was coding data from studies that used instruments that treat Individualism and Collectivism as independent constructs, often referred to as Idiocentrism and Allocentrism (e.g., Triandis, Leung, Villareal, \& Clack, 1985) or Independent and Interdependent Self-Construal (e.g., Matsumoto, 1999), and report two separate scores for each sub-dimension. Data from these studies accounted for $14.9 \%$ of data points for the Individualism 
dimension in our dataset. After carefully considering all relevant factors, we coded reported Individualism and Collectivism scores separately and then converted them to a single score by averaging the sum of the Individualism and the inverse of the Collectivism scores. Our decision was based on a careful review of available models of Individualism-Collectivism, including those of several influential scholars (e.g., Theodore Singelis, Harry Triandis) who proposed the bi-dimensional model of Individualism-Collectivism, and was guided by the following considerations. First, Hofstede's original model treats Individualism and Collectivism as the opposite ends of a single continuum. Second, the measure of Individualism in Hofstede's original VSM combines both Individualism and Collectivism items. It then converts the item scores into a single composite index by reverse-coding the Collectivism items, which is exactly what we did to convert our scores. Third, despite frequent arguments for theoretical independence of Individualism and Collectivism, empirical evidence generally shows that the two are indeed opposites of a single continuum as conceptualized by Hofstede. For example, based on a meta-analysis of the construct, Oyserman et al. (2002) reported that samples that scored high on Individualism tended to score low on Collectivism, and vice versa. Similarly, Taras, Sarala, and Muchinsky (2010) conducted a multitest investigation of the independence of Individualism and Collectivism based on information provided in 128 empirical papers and concluded that conceptually the two tend to represent the opposites of a single continuum. Finally, to validate our decision, we assessed convergence of the indices obtained from the studies that reported two separate scores for Individualism and Collectivism and those that treated the two as a single construct. The correlation was 0.75 , indicating functional equivalence.

After standardizing our data, we used the procedures described by Hunter and Schmidt (2004) for d-scores to derive our meta-analytic indices for each country/region along the four cultural dimensions. A d-score represents standardized mean difference between the observed post-treatment mean $\bar{X}_{o}$ and control or pre- and after-treatment mean $\bar{X}_{c}$ (1).

$$
d=\frac{X_{0}-X_{c}}{S D}
$$

In our case, however, we compare observed sample means not against control or pretreatment data, but against zero $\partial \mathrm{X}^{-} \mathrm{c}^{1 / 4} \mathrm{OP}$. Our data are standardized $(\mathrm{SD}=1)$. This way, our effect size is the extent of preference for certain cultural values $\partial \mathrm{d} 1 / 4 \mathrm{X}^{-} \mathrm{oP}$ with low numbers indicating low preference and high numbers indicating strong preference (e.g., low Power Distance vs. high Power Distance). The mean values from the samples representing the same country were used to estimate the country's score on the cultural dimensions. This is mathematically identical to aggregating individual responses to estimate national averages in conventional cross-cultural research. We weighted our national averages by sample size, but did not correct them for reliability attenuation as it is not applicable in our case, which is the aggregation of mean level data.

\subsection{Validation}

As our meta-analytic national culture indices are based on a different dataset, they naturally should differ from those of Hofstede's. Furthermore, we provide separate sets of indices for different decades, and it is expected that there will be decade-to-decade differences. 
Even though our dataset is larger and more representative, there remains the need to demonstrate whether our indices are more accurate than those offered by Hofstede and whether the decade-to decade variations are reflective of true culture changes and not measurement error. Thus, we validated our indices by testing them against several associated constructs and assumptions.

First, indices with a higher degree of validity should show stronger correlations with theoretically relevant economic and societal indicators. Consequently, to validate our indices, we need to demonstrate that there are substantive relationships between our cultural indices and other theoretically relevant variables. There is an unlimited array of factors that may be related to culture. To validate our indices, we selected only the criteria that have been repeatedly shown to correlate with cultural values of Hofstede's model and measures. These data were collected for the past 40 years by reputable sources. Specifically, we validated our indices against indicators or human development, income inequality, political freedom, corruption, urbanization rate, gross domestic product (GDP)/capita adjusted to purchasing power parity, divorce rate, occupants per household, gender equality, and innovation. Most of these factors have been hypothesized to relate to culture in Hofstede's (1980) Culture's Consequences. The relationships and their underlying theories have been discussed in great detail in many subsequent publications. There is universal agreement on the direction of the relationship and their causes (for recent reviews see Hofstede, 2001; House et al., 2004; Steel \& Taras, 2010; Taras, Kirkman, \& Steel, 2010). Therefore, we do not provide a detailed review of the theoretical rationale behind the relationships here but simply restate the commonly hypothesized relationships and their direction.

Power Distance relates to inequality and an accepted power differential. It has been shown to correlate with other measures that also reflect increased differences among people at various levels of the social hierarchy, such as income inequality, corruption, political freedom, and the human development index.

Individualism emphasizes independence and self-interest. It has been shown to be associated with economic independence, personal development, loyalty to family ties, and tendency to live in groups. We can assess these constructs with indicators such as: GDP/capita at PPP, human development index, urban population portion, divorce rate, number of occupants per household and percent of children who live with their parents.

Masculinity has two components, gender egalitarianism and achievement orientation. Thus, it correlates with such indicators as women's representation in national parliaments (i.e., gender egalitarianism) and the importance attributed to attributes of achievement such as good pay and promotion opportunities (i.e., achievement orientation). Furthermore, as masculinity relates to competition, work centrality, ability to cope with stress, and the desire for the latest technological advancements (Hofstede, 1980), it also has been shown to relate to achievement in science and technology as indicated by the rate of patent application per capita.

Uncertainty Avoidance is likely to be the most dynamic of the dimensions. Preference for clarity and certainty can change rapidly, reflecting the present economic and political stability. In times of crises, preference for stability and the need for clear rules and guidelines increases, such as what happened in the U.S. after the 9/11 attacks (Adams, 2005). Not surprisingly, Uncertainty Avoidance has been shown to correlate with such indicators of economic, political and societal problems such as inflation, corruption, criminal risk, and unemployment. It has also been theorized that Uncertainty Avoidance may hinder innovation, with observed correlations between the two at .45 at the national level (Taras, Kirkman, \& Steel, 2010). Thus, we expect to see negative correlations with patent application. 
To operationalize the variables used in this validation process, we used external data publicly available from the United Nations Organization (UNO, 2010), World Bank (WB, 2010), Freedom House (FH, 2010), and Heritage Foundation (HF, 2010) databases.

Second, if cultural change and Hofstede's indices deteriorate over time, then correlations between Hofstede's and our metaanalytic indices should be stronger for earlier decades (e.g., 1980s) than for later decades (e.g., 2000s). That is, the test-retest reliability should be decreasing over time.

Third, if cultures indeed change in a matter of decades, we should see the correlations between external national indicators representing a given decade to have the strongest correlations to cultural indices representing that same decade. Furthermore, the culture-criterion correlations should typically be higher for meta-analytic scores representing matching decades than for overall meta-analytic scores. However, we expect this to be a trend rather than a rule. Cultural values and external indicators, especially behaviors, can lag behind one another, such that the change in one later causes a change in the other (Taras, Steel, \& Kirkman, 2010). 
Table 1

National cultural scores, Hofstede's and meta-analytic (1970-2010 combined data)

\begin{tabular}{|c|c|c|c|c|c|c|c|c|c|c|c|c|c|c|c|c|}
\hline \multirow[t]{2}{*}{ Country/region } & \multicolumn{4}{|c|}{ Power Distance } & \multicolumn{4}{|c|}{ Individualism } & \multicolumn{4}{|l|}{ Masculinity } & \multicolumn{4}{|c|}{ Uncertainty Avoidance } \\
\hline & Hofstede's & $k$ & $N$ & Mean & Hofstede's & $k$ & $N$ & Mean & Hofstede's & $k$ & $N$ & Mean & Hofstede's & $k$ & $N$ & Mean \\
\hline Africa & 0.66 & 11 & 1145 & 1.08 & -1.00 & 31 & 2659 & -1.17 & -0.33 & 5 & 433 & -0.76 & -0.30 & 8 & 273 & 1.61 \\
\hline Arab countries & 1.08 & 16 & 1866 & 0.98 & -0.43 & 35 & 3207 & -0.67 & -0.09 & 18 & 1457 & 0.03 & 0.11 & 28 & 2499 & 0.63 \\
\hline Asian USSR & & 17 & 4343 & 1.04 & & 20 & 5518 & -0.30 & & 17 & 3922 & 0.92 & & 8 & 1564 & 0.70 \\
\hline Australia & -0.88 & 15 & 7234 & -0.64 & 1.70 & 35 & 9967 & 0.93 & 0.60 & 12 & 6729 & 0.61 & -0.38 & 9 & 6228 & -0.17 \\
\hline Austria & -1.99 & 4 & 1615 & -1.29 & 0.27 & 4 & 1492 & -0.07 & 1.55 & 5 & 1380 & 1.15 & 0.37 & 4 & 501 & -0.03 \\
\hline Baltic USSR & & 5 & 1182 & -0.66 & & 10 & 2303 & -0.32 & & 5 & 1182 & -0.19 & & 4 & 843 & 0.63 \\
\hline Belgium & 0.41 & 8 & 3188 & 0.37 & 1.09 & 14 & 3517 & 0.59 & 0.23 & 9 & 7582 & 0.19 & 1.30 & 7 & 1491 & 0.88 \\
\hline Bulgaria & & 1 & 177 & -0.37 & & 5 & 773 & -0.01 & & 3 & 607 & 0.32 & & 3 & 607 & 1.29 \\
\hline Canada & -0.74 & 22 & 19,532 & 0.02 & 1.29 & 59 & 8494 & 0.61 & 0.12 & 15 & 4552 & 0.24 & -0.49 & 15 & 4654 & -0.25 \\
\hline Caribbean & -0.47 & 17 & 1855 & -0.29 & -0.39 & 15 & 1325 & -0.59 & 0.97 & 1 & 77 & 0.93 & -1.86 & 11 & 1207 & -0.25 \\
\hline Central America & 0.78 & 18 & 5806 & 1.41 & -1.51 & 27 & 3476 & -1.14 & -0.44 & 25 & 3647 & 0.25 & 0.94 & 23 & 2969 & 1.08 \\
\hline China & 1.08 & 32 & 7012 & 0.71 & -1.17 & 60 & 9160 & -0.13 & 0.86 & 25 & 5102 & -0.44 & -1.20 & 23 & 4875 & 0.42 \\
\hline Czech Rep. & & 7 & 1270 & -0.47 & & 10 & 1829 & 0.08 & & 7 & 1270 & 0.39 & & 8 & 1357 & 0.24 \\
\hline Denmark & -1.68 & 6 & 1973 & -1.17 & 1.05 & 9 & 1771 & 0.48 & -1.78 & 11 & 2028 & -0.92 & -1.47 & 5 & 1616 & -1.31 \\
\hline Finland & -1.01 & 7 & 1514 & -0.90 & 0.60 & 7 & 1391 & 0.23 & -1.25 & 4 & 984 & -0.58 & -0.06 & 4 & 1049 & -0.03 \\
\hline France & 0.55 & 8 & 2022 & 0.41 & 0.92 & 17 & 12,847 & 0.39 & -0.35 & 17 & 12,173 & 0.06 & 0.99 & 8 & 1922 & 1.05 \\
\hline Germany & -0.92 & 20 & 19,214 & -0.49 & 0.76 & 26 & 18,413 & 0.30 & 0.86 & 22 & 19,417 & 0.64 & 0.17 & 17 & 19,833 & 0.43 \\
\hline Greece & 0.19 & 9 & 1262 & -0.12 & -0.55 & 12 & 878 & -0.72 & 0.39 & 8 & 1068 & 0.23 & 2.01 & 9 & 1423 & 1.29 \\
\hline Hong Kong & 0.55 & 20 & 2902 & 0.56 & -0.96 & 55 & 7893 & -0.19 & 0.39 & 25 & 2585 & 0.05 & -1.24 & 16 & 1879 & -0.37 \\
\hline Hungary & & 2 & 638 & 1.14 & & 3 & 326 & 0.11 & & 1 & 98 & 1.07 & & 2 & 290 & 1.07 \\
\hline India & 0.95 & 8 & 1152 & 0.83 & -0.02 & 38 & 3970 & -0.55 & 0.33 & 26 & 2568 & -0.18 & -0.81 & 19 & 2049 & -0.63 \\
\hline Indonesia & 0.99 & 4 & 153 & 0.69 & -1.41 & 10 & 628 & -0.58 & -0.20 & 5 & 197 & 0.13 & -0.49 & 7 & 155 & -0.58 \\
\hline Ireland & -1.23 & 6 & 1139 & -0.70 & 0.88 & 9 & 1806 & 0.42 & 0.97 & 8 & 1784 & 0.84 & -1.00 & 9 & 831 & -0.56 \\
\hline Israel & -1.90 & 6 & 850 & -0.95 & 0.23 & 11 & 1567 & 0.33 & -0.14 & 5 & 840 & 0.05 & 0.79 & 3 & 533 & 0.73 \\
\hline Italy & -0.25 & 10 & 3447 & -0.06 & 1.13 & 12 & 2842 & 0.49 & 1.07 & 12 & 3503 & 0.70 & 0.56 & 11 & 2845 & 0.62 \\
\hline Japan & -0.07 & 26 & 14,736 & 0.32 & -0.10 & 79 & 18,369 & -0.23 & 2.40 & 32 & 12,436 & 1.31 & 1.22 & 11 & 9387 & 1.33 \\
\hline Korea & 0.19 & 9 & 1034 & 0.69 & -1.25 & 49 & 6314 & -0.12 & -0.57 & 13 & 2018 & 0.45 & 0.95 & 10 & 1079 & 0.46 \\
\hline Malaysia & 2.15 & 9 & 875 & 1.38 & -0.92 & 18 & 1496 & -0.95 & 0.02 & 10 & 1934 & 0.11 & -0.96 & 8 & 968 & 0.32 \\
\hline Netherlands & -0.79 & 14 & 10,211 & -0.11 & 1.29 & 16 & 4574 & 0.89 & -1.89 & 9 & 2307 & -0.91 & -0.30 & 9 & 2310 & -0.27 \\
\hline New Zealand & -1.50 & 4 & 1513 & -0.87 & 1.25 & 8 & 2240 & 1.13 & 0.44 & 3 & 1431 & 0.10 & -0.45 & 4 & 1513 & -0.52 \\
\hline Norway & -1.10 & 10 & 1726 & -0.94 & 0.84 & 8 & 1800 & 0.57 & -2.21 & 12 & 1854 & -1.14 & -0.42 & 7 & 820 & -1.37 \\
\hline Philippines & 1.71 & 15 & 1787 & 1.14 & -0.68 & 13 & 1750 & -1.39 & 0.76 & 11 & 1125 & 0.26 & -0.65 & 12 & 1051 & 0.01 \\
\hline Poland & 0.55 & 6 & 1295 & -0.39 & 0.47 & 15 & 2694 & -0.33 & 0.76 & 9 & 1690 & 0.04 & 1.26 & 7 & 1382 & 0.54 \\
\hline Portugal & 0.33 & 3 & 494 & -0.14 & -0.88 & 12 & 1374 & -0.83 & -0.99 & 5 & 362 & -0.70 & 1.69 & 5 & 580 & 0.32 \\
\hline Romania & 1.53 & 4 & 986 & -0.20 & -0.76 & 8 & 1162 & -0.35 & -0.41 & 4 & 886 & -0.54 & 1.15 & 5 & 1198 & 0.75 \\
\hline Singapore & 0.82 & 10 & 912 & 0.79 & -1.17 & 32 & 4805 & -0.71 & -0.09 & 23 & 3437 & -0.19 & -2.06 & 9 & 905 & -0.65 \\
\hline Slavic USSR & & 19 & 5981 & -0.10 & & 33 & 12,726 & -0.25 & & 19 & 4411 & -0.05 & & 22 & 5614 & 1.12 \\
\hline South Africa & -0.30 & 7 & 1813 & 0.00 & 0.68 & 15 & 2742 & 0.53 & 0.70 & 16 & 2573 & -0.03 & -0.45 & 14 & 2626 & -0.21 \\
\hline South America & 0.33 & 46 & 27,178 & 1.17 & -0.75 & 54 & 13,203 & -0.73 & -0.15 & 51 & 11,773 & -0.52 & 0.87 & 39 & 10,177 & 0.94 \\
\hline Spain & 0.06 & 6 & 2279 & 0.16 & 0.10 & 9 & 2736 & 0.05 & -0.41 & 10 & 2646 & -0.13 & 0.99 & 6 & 2347 & 1.17 \\
\hline Sweden & -1.10 & 9 & 3515 & -0.76 & 0.92 & 9 & 3212 & 0.69 & -2.36 & 16 & 3574 & -0.95 & -1.24 & 8 & 3400 & -0.94 \\
\hline Switzerland & -0.96 & 7 & 2765 & -0.57 & 0.80 & 6 & 2560 & 0.40 & 1.07 & 7 & 2723 & 0.73 & -0.10 & 7 & 2595 & 0.14 \\
\hline Taiwan & 0.10 & 22 & 5888 & -0.23 & -1.29 & 49 & 7006 & -0.74 & -0.25 & 33 & 7107 & -0.21 & 0.33 & 18 & 5231 & -0.07 \\
\hline Thailand & 0.37 & 4 & 329 & 0.50 & -1.17 & 10 & 976 & -0.88 & -0.83 & 6 & 273 & -0.58 & 0.13 & 7 & 287 & 0.16 \\
\hline Turkey & 0.46 & 8 & 707 & 0.09 & -0.47 & 21 & 3953 & -0.39 & -0.25 & 12 & 1190 & 0.37 & 0.95 & 5 & 503 & 0.41 \\
\hline UK & -0.92 & 31 & 37,820 & 0.03 & 1.66 & 32 & 11,743 & 0.93 & 0.86 & 22 & 8794 & 0.83 & -1.00 & 24 & 10,339 & -0.61 \\
\hline USA & -0.70 & 132 & 38,843 & -0.27 & 1.74 & 552 & 78,701 & 0.42 & 0.65 & 149 & 28,509 & 0.20 & -0.57 & 109 & 28,767 & -0.14 \\
\hline Vietnam & 0.64 & & & & -1.17 & 2 & 174 & -0.46 & -0.51 & 1 & 22 & -0.46 & -1.20 & 3 & 24 & -0.12 \\
\hline Yugoslavia & 0.90 & 3 & 1760 & 0.29 & -0.88 & 4 & 1762 & -0.03 & -1.52 & 2 & 996 & -0.73 & 1.07 & 3 & 1760 & 0.22 \\
\hline Total $k, N$ & & 683 & 256,938 & & & 1588 & 296,124 & & & 771 & 189,280 & & & 613 & 158,364 & \\
\hline
\end{tabular}

Note: $k$, number of samples in the meta-analytic dataset; $N$, total number of individuals. Hofstede's scores are standardized to make them comparable with meta-analytic scores. 
Table 2

Meta-analytic national Power Distance scores.

\begin{tabular}{|c|c|c|c|c|c|c|c|c|c|}
\hline \multirow[t]{2}{*}{ Country/region } & \multicolumn{3}{|c|}{$1980 \mathrm{~s}$} & \multicolumn{3}{|c|}{$1990 \mathrm{~s}$} & \multicolumn{3}{|c|}{$2000 \mathrm{~s}$} \\
\hline & $k$ & $N$ & Score & $k$ & $N$ & Score & $k$ & $N$ & Score \\
\hline Africa & & & & 9 & 943 & 1.20 & 2 & 202 & 0.48 \\
\hline Arab countries & 8 & 1142 & 1.06 & 4 & 355 & 1.03 & 4 & 369 & 0.67 \\
\hline Asian USSR & & & & 7 & 1541 & 1.20 & 10 & 2802 & 0.96 \\
\hline Australia & 1 & 1919 & -0.38 & 11 & 5028 & -0.73 & 3 & 287 & -0.79 \\
\hline Austria & 4 & 1615 & -1.29 & & & & & & \\
\hline Baltic USSR & & & & 4 & 1019 & -0.63 & 1 & 163 & -0.84 \\
\hline Belgium & 4 & 2732 & 0.50 & & & & 4 & 456 & -0.40 \\
\hline Bulgaria & & & & & & & 1 & 177 & -0.37 \\
\hline Canada & 10 & 18,250 & 0.06 & 5 & 342 & -0.44 & 7 & 940 & -0.56 \\
\hline Caribbean & 1 & 201 & 0.28 & 4 & 287 & -0.08 & 12 & 1367 & -0.42 \\
\hline Central America & 9 & 5448 & 1.61 & 8 & 940 & 0.59 & 9 & 336 & 0.50 \\
\hline China & 7 & 1427 & 0.82 & 14 & 3407 & 0.99 & 11 & 2178 & 0.18 \\
\hline Czech Rep. & & & & 2 & 524 & -0.64 & 5 & 746 & -0.34 \\
\hline Denmark & 4 & 1644 & -1.25 & 2 & 329 & -0.81 & & & \\
\hline Finland & 4 & 1163 & -0.94 & 1 & 117 & -0.81 & 2 & 234 & -0.80 \\
\hline France & 4 & 1641 & 0.58 & 3 & 321 & -0.21 & 1 & 60 & -0.70 \\
\hline Germany & 7 & 13,256 & -0.46 & 8 & 2698 & -0.44 & 5 & 3260 & -0.77 \\
\hline Greece & 5 & 531 & 0.10 & 3 & 653 & -0.35 & 1 & 78 & 0.36 \\
\hline Hong Kong & 4 & 769 & 1.98 & 11 & 845 & 0.24 & 5 & 1288 & -0.09 \\
\hline Hungary & 1 & 540 & 1.34 & & & & 1 & 98 & 0.03 \\
\hline India & 4 & 794 & 1.17 & 3 & 202 & 0.35 & 1 & 156 & -0.28 \\
\hline Indonesia & 1 & 55 & 1.17 & 3 & 98 & 0.42 & & & \\
\hline Ireland & 4 & 419 & -1.07 & 2 & 720 & -0.49 & & & \\
\hline Israel & 2 & 417 & -1.13 & & & & 4 & 433 & -0.78 \\
\hline Italy & 8 & 2446 & -0.02 & 2 & 1001 & -0.15 & & & \\
\hline Japan & 7 & 10,993 & 0.56 & 14 & 1687 & 0.14 & 5 & 2056 & -0.97 \\
\hline Korea & 2 & 150 & 1.12 & 7 & 884 & 0.61 & & & \\
\hline Malaysia & 1 & 55 & 2.13 & 7 & 728 & 1.47 & 1 & 92 & 0.21 \\
\hline Netherlands & 9 & 9935 & -0.09 & 2 & 124 & -0.47 & 3 & 152 & -1.33 \\
\hline New Zealand & 1 & 413 & -0.90 & 2 & 567 & -0.68 & 1 & 533 & -1.04 \\
\hline Norway & 4 & 1134 & -1.03 & 5 & 571 & -0.73 & 1 & 21 & -1.38 \\
\hline Philippines & 7 & 950 & 1.85 & 3 & 329 & 0.48 & 5 & 508 & 0.23 \\
\hline Poland & & & & 4 & 931 & -0.47 & 2 & 364 & -0.17 \\
\hline Portugal & 3 & 494 & -0.14 & & & & & & \\
\hline Romania & & & & 2 & 406 & 0.34 & 2 & 580 & -0.58 \\
\hline Singapore & 1 & 58 & 1.02 & 9 & 854 & 0.77 & & & \\
\hline Slavic USSR & & & & 13 & 2983 & 0.20 & 6 & 2998 & -0.39 \\
\hline South Africa & 1 & 867 & 0.10 & 4 & 622 & -0.09 & 2 & 324 & -0.07 \\
\hline South America & 21 & 21,884 & 1.36 & 11 & 4458 & 0.62 & 14 & 836 & -0.70 \\
\hline Spain & 4 & 1946 & 0.30 & & & & 2 & 333 & -0.67 \\
\hline Sweden & 4 & 2751 & -0.71 & 2 & 460 & -0.86 & 3 & 304 & -1.14 \\
\hline Switzerland & 4 & 2414 & -0.59 & 2 & 309 & -0.39 & 1 & 42 & -0.48 \\
\hline Taiwan & 5 & 2950 & -0.04 & 11 & 1799 & -0.46 & 6 & 1139 & -0.37 \\
\hline Thailand & 1 & 80 & 0.65 & 3 & 249 & 0.45 & & & \\
\hline Turkey & 5 & 490 & 0.13 & 2 & 68 & 0.10 & 1 & 149 & -0.06 \\
\hline UK & 16 & 35,926 & 0.06 & 11 & 1536 & -0.49 & 4 & 358 & -0.81 \\
\hline USA & 22 & 6384 & -0.14 & 74 & 26,209 & -0.27 & 36 & 6250 & -0.39 \\
\hline Vietnam & & & & & & & & & \\
\hline Yugoslavia & 1 & 248 & 1.09 & 2 & 1512 & 0.16 & & & \\
\hline Average & 5 & 3913 & 0.27 & 7 & 1693 & 0.00 & 5 & 860 & -0.37 \\
\hline Total $k, N$; score SD & 211 & 156,531 & 0.94 & 288 & 61,716 & 0.64 & 184 & 32,669 & 0.55 \\
\hline
\end{tabular}

Note: $\mathrm{k}$, number of samples in the meta-analytic dataset; $N$, total number of individuals. 
Table 3

Meta-analytic national Individualism scores.

\begin{tabular}{|c|c|c|c|c|c|c|c|c|c|}
\hline \multirow[t]{2}{*}{ Country/region } & \multicolumn{3}{|c|}{$1980 \mathrm{~s}$} & \multicolumn{3}{|c|}{$1990 \mathrm{~s}$} & \multicolumn{3}{|c|}{$2000 \mathrm{~s}$} \\
\hline & $k$ & $N$ & Score & $k$ & $N$ & Score & $k$ & $N$ & Score \\
\hline Africa & 2 & 214 & -1.38 & 17 & 1615 & -1.49 & 12 & 830 & -0.50 \\
\hline Arab countries & 15 & 1108 & -0.38 & 10 & 1411 & -0.76 & 10 & 688 & -0.94 \\
\hline Asian USSR & & & & 7 & 1541 & -0.36 & 13 & 3977 & -0.27 \\
\hline Australia & 3 & 1125 & 0.83 & 21 & 7444 & 0.93 & 11 & 1398 & 0.98 \\
\hline Austria & 4 & 1492 & -0.07 & & & & & & \\
\hline Baltic USSR & & & & 9 & 2140 & -0.35 & 1 & 163 & 0.06 \\
\hline Belgium & 4 & 2609 & 0.56 & 4 & 294 & 0.69 & 6 & 614 & 0.67 \\
\hline Bulgaria & & & & 4 & 596 & -0.05 & 1 & 177 & 0.13 \\
\hline Canada & 6 & 3946 & 0.62 & 23 & 1986 & 0.37 & 30 & 2562 & 0.78 \\
\hline Caribbean & 3 & 81 & -0.81 & & & & 12 & 1244 & -0.58 \\
\hline Central America & 5 & 1271 & -1.24 & 13 & 1020 & -1.26 & 9 & 1185 & -1.03 \\
\hline China & 6 & 342 & -0.57 & 38 & 6070 & -0.19 & 16 & 2748 & 0.02 \\
\hline Czech Rep. & & & & 5 & 1083 & -0.13 & 5 & 746 & 0.39 \\
\hline Denmark & 4 & 1521 & 0.52 & 1 & 86 & 0.47 & 4 & 164 & 0.77 \\
\hline Finland & 4 & 1040 & 0.32 & 3 & 351 & -0.04 & & & \\
\hline France & 6 & 11,917 & 0.39 & 6 & 462 & 0.18 & 5 & 468 & 0.60 \\
\hline Germany & 6 & 12,059 & 0.26 & 11 & 2392 & 0.31 & 9 & 3962 & 0.40 \\
\hline Greece & 6 & 546 & -0.99 & 3 & 97 & -0.73 & 3 & 235 & -0.08 \\
\hline Hong Kong & 11 & 1255 & -0.99 & 35 & 4083 & -0.12 & 9 & 2555 & 0.09 \\
\hline Hungary & & & & 2 & 228 & 0.18 & 1 & 98 & -0.06 \\
\hline India & 10 & 1133 & -1.09 & 17 & 1352 & -0.42 & 11 & 1485 & -0.28 \\
\hline Indonesia & 3 & 278 & -1.31 & 7 & 350 & 0.01 & & & \\
\hline Ireland & 4 & 396 & 0.47 & 1 & 300 & 0.35 & 4 & 1110 & 0.42 \\
\hline Israel & 3 & 795 & -0.27 & 4 & 289 & 0.98 & 4 & 483 & 0.89 \\
\hline Italy & 8 & 2500 & 0.56 & 3 & 229 & -0.13 & 1 & 113 & 0.32 \\
\hline Japan & 4 & 7089 & -0.52 & 49 & 7101 & -0.14 & 26 & 4179 & 0.11 \\
\hline Korea & 1 & 78 & -1.58 & 34 & 4234 & -0.37 & 14 & 2002 & 0.45 \\
\hline Malaysia & 1 & 78 & -1.29 & 10 & 681 & -0.95 & 7 & 737 & -0.93 \\
\hline Netherlands & 7 & 2721 & 0.77 & 3 & 125 & 0.94 & 6 & 1728 & 1.07 \\
\hline New Zealand & 1 & 413 & 0.69 & 4 & 1065 & 1.37 & 3 & 762 & 1.03 \\
\hline Norway & 4 & 1011 & 0.49 & 2 & 734 & 0.65 & 2 & 55 & 0.95 \\
\hline Philippines & 6 & 631 & -1.38 & 7 & 1119 & -1.18 & & & \\
\hline Poland & & & & 9 & 1818 & -0.37 & 6 & 876 & -0.24 \\
\hline Portugal & 1 & 243 & -1.25 & 5 & 447 & -0.38 & 6 & 684 & -0.22 \\
\hline Romania & & & & 6 & 682 & -0.30 & 2 & 480 & -0.44 \\
\hline Singapore & 2 & 115 & -1.09 & 20 & 3555 & -0.97 & 10 & 1135 & 0.19 \\
\hline Slavic USSR & & & & 21 & 5656 & -0.68 & 12 & 7070 & 0.09 \\
\hline South Africa & 1 & 867 & 0.17 & 4 & 568 & 0.37 & 10 & 1307 & 0.83 \\
\hline South America & 11 & 6347 & -0.98 & 20 & 4401 & -0.71 & 23 & 2455 & -0.12 \\
\hline Spain & 4 & 2005 & -0.22 & 1 & 68 & 0.51 & 4 & 663 & 0.83 \\
\hline Sweden & 5 & 2682 & 0.49 & 2 & 227 & 1.54 & 2 & 303 & 1.79 \\
\hline Switzerland & 4 & 2260 & 0.33 & & & & 2 & 300 & 0.93 \\
\hline Taiwan & 11 & 1380 & -0.95 & 19 & 2471 & -0.46 & 19 & 3155 & -0.47 \\
\hline Thailand & 1 & 80 & -1.51 & 4 & 127 & -1.34 & 5 & 769 & -0.73 \\
\hline Turkey & 2 & 246 & -0.53 & 9 & 724 & -0.30 & 10 & 2983 & -0.45 \\
\hline UK & 9 & 7821 & 1.00 & 18 & 3622 & 0.82 & 5 & 300 & 0.33 \\
\hline USA & 42 & 10,281 & 0.69 & 348 & 44,376 & 0.37 & 162 & 24,044 & 0.39 \\
\hline Vietnam & & & & 1 & 22 & -1.00 & 1 & 152 & 0.41 \\
\hline Yugoslavia & 1 & 248 & -1.25 & 3 & 1514 & 0.20 & & & \\
\hline Average & 6 & 2306 & -0.31 & 18 & 2625 & -0.09 & 12 & 1890 & 0.18 \\
\hline Total $k, N$; score SD & 231 & 92,224 & 0.82 & 843 & 120,756 & 0.71 & 514 & 83,144 & 0.63 \\
\hline
\end{tabular}

Note: $k$, number of samples in the meta-analytic dataset; $N$, total number of individuals.

\section{Results}

\subsection{Sample}

The final pool contained 451 journal articles, Master's and doctoral theses, books and book chapters, and unpublished papers coming from five continents and written in eight languages. Most studies were based on multi-sample designs. The number of reported sample means per dimension varied from 613 (representing 158,364 respondents) for Uncertainty Avoidance to 1588 (representing to 296,124 respondents) for Individualism. The vast majority of the samples consisted of middle or upper middle-class people of working age (overall mean 33.4 years), with a roughly even gender structure (male 47.4\%) and fairly well educated (overall mean 15.5 years of schooling). The demographics did not vary noticeably across countries, with the 
exception of samples from Arab countries that had a somewhat higher percentage of men, which actually better represented the composition of the labor force in the region.

\subsection{Cultural indices and their validity}

Table 1 reports both Hofstede's $(1980,2001)$ cultural scores and our overall metaanalytically derived ones. Note, to enable direct comparison, Hofstede's scores were standardized and, in some cases, regrouped for matching regions. The main result of this study is presented in Tables 2-5. The tables provide our metaanalytic scores parsed by decade for each of the four cultural dimensions. The indices can be used to study the effects of cultural dynamics overall and by country as well as to explore the relationship between culture and other factors. It must be noted, however, that the reliability of indices for different countries varies due to differences in the number of the original data points behind each meta-analytic effect size. Therefore, the tables also provide $\mathrm{N}$ (number of individuals) and $\mathrm{k}$ (number of studies) representing each index to aid more accurate interpretation of the data presented.

Although the reported meta-analytic indices are overall consistent with those reported by Hofstede (1980), some nations deviate from their positions in ranking tables in Hofstede's report. The difference is especially noticeable for more recent decades. For example, many Eastern European and South American countries, generally described as comparatively high Power Distance oriented and Collectivist, had lower scores on Power Distance and higher scores on Individualism, in particular for the 1990s and 2000s, than what would be expected based on Hofstede's study. Conversely, the U.S. and some other Western nations, such as Canada and Germany, scored much lower on Individualism and higher on Power Distance compared to their scores reported by Hofstede.

To test whether these inconsistencies are a result of problems with our methodology or whether they reflect cultural change, we conducted a validation analysis by comparing metaanalytic cultural indices against those offered by Hofstede, as well as checked how the indices correlate with theoretically relevant external criteria (Tables 6-9). First, a comparison against Hofstede's original indices provides initial validation for our meta-analytic cultural indices (Tables 6-9, first row). Overall meta-analytically derived scores match those of Hofstede quite closely with correlations of 0.85 for Power Distance, 0.86 for Individualism, 0.85 for Masculinity, and 0.72 for Uncertainty Avoidance. Although these correlations are relatively high, the differences become noticeable when we compare Hofstede's scores to meta-analytic scores reported by decade. These relationships drop to as low as 0.46 , as is the case for the correlation between Hofstede's and meta-analytic Uncertainty Avoidance score for the 2000s. Do these differences reflect changes in sampling, changing culture, or possibly the effects of sampling error and methodology artifacts? To answer these questions, we performed a series of validity checks as planned in Section 3. 
Table 4

Meta-analytic national Masculinity scores.

\begin{tabular}{|c|c|c|c|c|c|c|c|c|c|}
\hline \multirow[t]{2}{*}{ Country/region } & \multicolumn{3}{|c|}{$1980 \mathrm{~s}$} & \multicolumn{3}{|c|}{$1990 \mathrm{~s}$} & \multicolumn{3}{|c|}{$2000 s$} \\
\hline & $k$ & $N$ & Score & $k$ & $N$ & Score & $k$ & $N$ & Score \\
\hline Africa & & & & 5 & 433 & -0.76 & & & \\
\hline Arab countries & 7 & 797 & -0.04 & 3 & 167 & 0.52 & 8 & 493 & 0.23 \\
\hline Asian USSR & & & & 7 & 1541 & 0.60 & 10 & 2381 & 1.13 \\
\hline Australia & 1 & 1919 & 0.69 & 8 & 4418 & 0.56 & 3 & 398 & 0.50 \\
\hline Austria & 5 & 1380 & 1.15 & & & & & & \\
\hline Baltic USSR & & & & 4 & 1019 & -0.26 & 1 & 163 & 0.26 \\
\hline Belgium & 6 & 7323 & 0.25 & 1 & 53 & 0.35 & 2 & 206 & -1.05 \\
\hline Bulgaria & & & & 2 & 430 & 0.49 & 1 & 177 & -0.11 \\
\hline Canada & 6 & 3946 & 0.36 & 6 & 487 & -0.68 & 3 & 119 & -0.29 \\
\hline Caribbean & 1 & 77 & 0.93 & & & & & & \\
\hline Central America & 6 & 1822 & 0.70 & 7 & 1051 & -0.38 & 12 & 774 & 0.05 \\
\hline China & 5 & 1276 & -0.67 & 12 & 2394 & -0.35 & 8 & 1432 & -0.39 \\
\hline Czech Rep. & & & & 2 & 524 & 0.68 & 5 & 746 & 0.19 \\
\hline Denmark & 6 & 1531 & -1.01 & 3 & 415 & -0.18 & 2 & 82 & -1.23 \\
\hline Finland & 2 & 862 & -0.55 & 2 & 122 & -0.74 & & & \\
\hline France & 9 & 11,624 & 0.06 & 2 & 120 & -0.55 & 6 & 429 & 0.40 \\
\hline Germany & 11 & 11,898 & 0.81 & 6 & 5857 & 0.56 & 6 & 1670 & -0.33 \\
\hline Greece & 5 & 352 & 0.09 & 2 & 600 & 0.60 & 2 & 119 & -1.20 \\
\hline Hong Kong & 4 & 769 & -0.04 & 17 & 1121 & 0.24 & 5 & 698 & -0.13 \\
\hline Hungary & & & & & & & 1 & 98 & 1.07 \\
\hline India & 2 & 308 & 0.39 & 19 & 1813 & -0.22 & 5 & 447 & -0.40 \\
\hline Indonesia & 1 & 77 & 0.18 & 4 & 120 & 0.10 & & & \\
\hline Ireland & 1 & 251 & 0.93 & 1 & 420 & 0.45 & 6 & 1113 & 0.97 \\
\hline Israel & 1 & 357 & 0.21 & & & & 4 & 483 & -0.07 \\
\hline Italy & 8 & 2388 & 0.69 & 2 & 1001 & 0.75 & 2 & 114 & 0.53 \\
\hline Japan & 9 & 8220 & 1.73 & 10 & 1063 & 0.91 & 13 & 3153 & 0.34 \\
\hline Korea & 4 & 480 & 0.78 & 7 & 1194 & 0.31 & 2 & 344 & 0.46 \\
\hline Malaysia & 1 & 77 & 0.31 & 5 & 1416 & 0.17 & 5 & 442 & -0.11 \\
\hline Netherlands & 3 & 1863 & -0.96 & 4 & 294 & -0.62 & 2 & 150 & -0.87 \\
\hline New Zealand & 1 & 413 & 0.59 & 2 & 1018 & -0.09 & & & \\
\hline Norway & 6 & 1057 & -1.35 & 5 & 776 & -0.85 & 1 & 21 & -0.89 \\
\hline Philippines & 6 & 631 & 0.39 & 5 & 494 & 0.09 & & & \\
\hline Poland & & & & 5 & 1060 & 0.12 & 5 & 633 & -0.10 \\
\hline Portugal & 5 & 362 & -0.64 & & & & & & \\
\hline Romania & & & & 2 & 406 & -0.49 & 2 & 480 & -0.58 \\
\hline Singapore & 2 & 115 & 0.47 & 15 & 2724 & -0.10 & 6 & 598 & -0.74 \\
\hline Slavic USSR & & & & 16 & 4270 & -0.07 & 3 & 141 & 0.40 \\
\hline South Africa & 3 & 914 & 0.35 & 3 & 352 & 0.21 & 10 & 1307 & -0.14 \\
\hline South America & 13 & 5798 & -0.34 & 10 & 3062 & -0.44 & 28 & 2913 & -0.97 \\
\hline Spain & 6 & 2015 & -0.12 & & & & 4 & 631 & -0.18 \\
\hline Sweden & 12 & 3011 & -0.83 & 3 & 352 & -1.05 & 1 & 211 & -1.46 \\
\hline Switzerland & 4 & 2290 & 0.88 & 2 & 391 & -0.05 & 1 & 42 & -0.04 \\
\hline Taiwan & 6 & 3491 & -0.49 & 20 & 2687 & 0.09 & 7 & 929 & -0.01 \\
\hline Thailand & 1 & 80 & -0.24 & 5 & 193 & -0.64 & & & \\
\hline Turkey & 5 & 311 & -0.20 & & & & 7 & 879 & 0.57 \\
\hline UK & 8 & 7341 & 0.92 & 9 & 1231 & 0.37 & 5 & 222 & 0.58 \\
\hline USA & 20 & 6690 & 0.72 & 88 & 12,594 & 0.05 & 41 & 9225 & 0.04 \\
\hline Vietnam & & & & 1 & 22 & -0.48 & & & \\
\hline Yugoslavia & 1 & 248 & -0.68 & 1 & 748 & -0.74 & & & \\
\hline Average & 5 & 2420 & 0.16 & 8 & 1439 & -0.04 & 6 & 907 & -0.09 \\
\hline Total $k, N$; score SD & 203 & 94,364 & 0.68 & 333 & 60,453 & 0.51 & 235 & 34,463 & 0.64 \\
\hline
\end{tabular}

Note: $k$, number of samples in the meta-analytic dataset; $N$, total number of individuals. 
Table 5

Meta-analytic national Uncertainty Avoidance scores.

\begin{tabular}{|c|c|c|c|c|c|c|c|c|c|}
\hline \multirow[t]{2}{*}{ Country/region } & \multicolumn{3}{|c|}{$1980 \mathrm{~s}$} & \multicolumn{3}{|c|}{$1990 \mathrm{~s}$} & \multicolumn{3}{|c|}{$2000 \mathrm{~s}$} \\
\hline & $k$ & $N$ & Score & $k$ & $N$ & Score & $k$ & $N$ & Score \\
\hline Africa & & & & 3 & 267 & 1.63 & 5 & 6 & 1.03 \\
\hline Arab countries & 11 & 1421 & 0.76 & 4 & 534 & 0.78 & 13 & 544 & 0.16 \\
\hline Asian USSR & & & & 7 & 1541 & 0.69 & 1 & 23 & 1.60 \\
\hline Australia & 1 & 1919 & -0.07 & 6 & 4206 & -0.21 & 2 & 103 & -0.47 \\
\hline Austria & 4 & 501 & -0.03 & & & & & & \\
\hline Baltic USSR & & & & 3 & 680 & 0.77 & 1 & 163 & 0.03 \\
\hline Belgium & 4 & 1032 & 0.97 & & & & 3 & 459 & 0.67 \\
\hline Bulgaria & & & & 2 & 430 & 1.63 & 1 & 177 & 0.46 \\
\hline Canada & 6 & 3946 & -0.25 & 4 & 297 & -0.48 & 5 & 411 & -0.01 \\
\hline Caribbean & 1 & 87 & -1.65 & & & & 10 & 1120 & -0.14 \\
\hline Central America & 6 & 1880 & 1.33 & 8 & 731 & 0.80 & 9 & 358 & 0.39 \\
\hline China & 6 & 1319 & 1.08 & 11 & 3036 & 0.22 & 6 & 520 & -0.08 \\
\hline Czech Rep. & & & & 3 & 611 & 0.15 & 5 & 746 & 0.31 \\
\hline Denmark & 4 & 1530 & -1.36 & 1 & 86 & -0.58 & & & \\
\hline Finland & 4 & 1049 & -0.03 & & & & & & \\
\hline France & 5 & 1742 & 1.08 & 3 & 180 & 0.76 & & & \\
\hline Germany & 5 & 11,721 & 0.48 & 6 & 6536 & 0.52 & 6 & 1576 & -0.32 \\
\hline Greece & 5 & 417 & 1.51 & 2 & 1000 & 1.20 & 2 & 6 & 0.70 \\
\hline Hong Kong & 4 & 769 & -0.34 & 9 & 743 & -0.53 & 3 & 375 & -0.10 \\
\hline Hungary & & & & 1 & 192 & 1.27 & 1 & 98 & 0.69 \\
\hline India & 2 & 266 & -0.29 & 16 & 1627 & -0.74 & 1 & 156 & -0.07 \\
\hline Indonesia & 1 & 23 & -0.19 & 4 & 120 & -0.68 & 2 & 12 & -0.28 \\
\hline Ireland & 4 & 405 & -0.86 & 1 & 420 & -0.27 & 4 & 6 & 0.14 \\
\hline Israel & 1 & 357 & 1.19 & & & & 2 & 176 & -0.19 \\
\hline Italy & 8 & 2318 & 0.71 & 1 & 517 & 0.23 & 2 & 10 & -0.10 \\
\hline Japan & 3 & 6930 & 1.71 & 3 & 407 & 0.32 & 5 & 2050 & 0.24 \\
\hline Korea & 1 & 78 & 1.35 & 7 & 935 & 0.46 & 2 & 66 & -0.53 \\
\hline Malaysia & 1 & 22 & -0.69 & 5 & 944 & 0.34 & 2 & 2 & -0.64 \\
\hline Netherlands & 4 & 2118 & -0.22 & 1 & 38 & -0.48 & 4 & 154 & -0.83 \\
\hline New Zealand & 1 & 413 & -0.15 & 2 & 567 & -0.22 & 1 & 533 & -1.13 \\
\hline Norway & 4 & 282 & -0.97 & 2 & 517 & -1.59 & 1 & 21 & -1.49 \\
\hline Philippines & 6 & 631 & 0.21 & 4 & 408 & -0.30 & 2 & 12 & 0.11 \\
\hline Poland & & & & 5 & 1018 & 0.56 & 2 & 364 & 0.48 \\
\hline Portugal & 5 & 580 & 0.32 & & & & & & \\
\hline Romania & & & & 3 & 493 & 1.33 & 2 & 705 & 0.34 \\
\hline Singapore & 1 & 58 & -1.86 & 7 & 806 & -0.54 & 1 & 41 & -1.19 \\
\hline Slavic USSR & & & & 17 & 5343 & 1.13 & 5 & 271 & 1.10 \\
\hline South Africa & 1 & 867 & -0.15 & 3 & 452 & -0.10 & 10 & 1307 & -0.29 \\
\hline South America & 9 & 5779 & 1.25 & 9 & 3577 & 0.42 & 21 & 821 & 1.00 \\
\hline Spain & 4 & 2014 & 1.18 & & & & 2 & 333 & 1.12 \\
\hline Sweden & 4 & 2637 & -1.07 & 2 & 460 & -0.09 & 2 & 303 & -1.13 \\
\hline Switzerland & 4 & 2300 & 0.15 & 1 & 211 & 0.02 & 2 & 84 & 0.33 \\
\hline Taiwan & 5 & 2988 & 0.20 & 7 & 1580 & -0.13 & 6 & 663 & -1.15 \\
\hline Thailand & 1 & 80 & 0.48 & 4 & 169 & 0.10 & 2 & 38 & -0.27 \\
\hline Turkey & 3 & 340 & 0.55 & & & & 2 & 163 & 0.11 \\
\hline UK & 9 & 8617 & -0.66 & 8 & 1211 & -0.43 & 7 & 511 & -0.22 \\
\hline USA & 35 & 7981 & -0.05 & so & 17,963 & -0.17 & 24 & 2823 & -0.18 \\
\hline Vietnam & & & & 1 & 22 & -0.18 & 2 & 2 & 0.61 \\
\hline Yugoslavia & 1 & 248 & 1.48 & 2 & 1512 & 0.02 & & & \\
\hline Average & 5 & 1991 & 0.23 & 6 & 1522 & 0.19 & 4 & 426 & 0.02 \\
\hline Total $k, N$; score SD & 184 & 77,665 & 0.87 & 238 & 62,387 & 0.69 & 191 & 18,312 & 0.68 \\
\hline
\end{tabular}

Note: $k$, number of samples in the meta-analytic dataset; $N$, total number of individuals.

Second, we confirmed the construct validity of the indices. The data in Tables 6-9 reveal that both Hofstede's scores and the metaanalytic national cultural scores correlate with the theoretically relevant external criteria. All of these correlations are in the expected direction and most at a statistically significant level (generally correlations exceeding .30 are significant at $p<$ .05). Our meta-analytic scores also tended to outperform Hofstede's scores. The theoretically relevant indicators correlated the same or stronger with the meta-analytic scores than with the scores from the IBM study in over two-thirds of the cases, or, specifically, in 58\% cases for Power Distance (Table 6), 67\% for Individualism (Table 7), 42\% for Masculinity (Table 8), and 89\% for Uncertainty Avoidance (Table 9).

Third, we calculated the test-retest reliability of Hofstede's scores (correlations between Hofstede's scores and corresponding meta-analytic scores for each decade) to determine if they have been decreasing as this would indicate cultural change. For all four dimensions, the correlations between Hofstede's and the metaanalytic scores are indeed diminishing over time 
(see data in Tables 6-9). Hofstede's scores always have the strongest correlations with the metaanalytic scores of the 1980s and the weakest with the scores representing the 2000s. For example, Hofstede's masculinity score decreases from 0.85 in the 1980 s to 0.56 in the $2000 \mathrm{~s}$. At this present rate of decline, none of Hofstede's scores will have a recognizable connection to the world's culture by 2050 with some, such as masculinity, probably becoming fully disconnected between 2020 and 2030.

Furthermore, the criterion validity of Hofstede's scores tends to deteriorate over time as well. For Individualism and Masculinity, the correlations between Hofstede's indices and the criteria representing the 1970/80s are quite strong. The correlation decline with each passing decade from an average of about 0.70 with the criteria representing the 1970/80s to about 0.60 with criteria representing the 1990 s and to about 0.50 with criteria representing the $2000 \mathrm{~s}$ (Tables 7 and 8). The decline is not as dramatic for Power Distance, but it is also noticeable for most criteria (Table 6). Interestingly, the relationship with Hofstede's scores and income inequality appears to be increasing over time, indicating that this may be a case where practices lag behind values. Finally, Uncertainty Avoidance shows a downward trend for about half of the criteria, but correlations with one criteria, Unemployment, actually show an increasing trend and correlations with the other two criteria are not linear with the trend changing from decade to decade (Table 9). Given that Uncertainty Avoidance is more capable of rapid change relative to the other values (e.g., Adams, 2005), it appears that this cultural value does indeed quickly shift and can abruptly change direction.

\begin{tabular}{|c|c|c|c|c|c|}
\hline \multirow[t]{3}{*}{ Indicators } & \multicolumn{5}{|c|}{ Culture scores } \\
\hline & \multirow{2}{*}{$\begin{array}{l}\text { Hofstede's } \\
\text { scores }\end{array}$} & \multicolumn{4}{|c|}{ Meta-analytic scores } \\
\hline & & Overall & $1980 \mathrm{~s}$ & $1990 \mathrm{~s}$ & $2000 s$ \\
\hline $\begin{array}{l}\text { Hofstede's Power } \\
\text { Distance scores }\end{array}$ & 1.00 & 0.85 & 0.91 & 0.83 & 0.70 \\
\hline \multicolumn{6}{|c|}{ Human development index } \\
\hline $1970 / 80 \mathrm{~s}$ & -0.63 & -0.63 & -0.61 & -0.72 & -0.59 \\
\hline $1990 \mathrm{~s}$ & -0.59 & -0.63 & -0.58 & -0.70 & -0.54 \\
\hline $2000 s$ & -0.58 & -0.55 & -0.54 & -0.65 & -0.62 \\
\hline \multicolumn{6}{|c|}{ Income inequality, GINI } \\
\hline $1970 / 80 \mathrm{~s}$ & 0.29 & 0.45 & 0.40 & 0.51 & 0.42 \\
\hline $1990 \mathrm{~s}$ & 0.31 & 0.44 & 0.40 & 0.47 & 0.42 \\
\hline $2000 s$ & 0.42 & 0.54 & 0.48 & 0.66 & 0.65 \\
\hline \multicolumn{6}{|l|}{ Political freedom } \\
\hline $1970 / 80 \mathrm{~s}$ & -0.70 & -0.53 & -0.65 & -0.71 & -0.61 \\
\hline $1990 \mathrm{~s}$ & -0.70 & -0.67 & -0.68 & -0.84 & -0.74 \\
\hline $2000 s$ & -0.66 & -0.66 & -0.67 & -0.85 & -0.76 \\
\hline \multicolumn{6}{|c|}{ Corruption perception index } \\
\hline $1970 / 80 \mathrm{~s}$ & 0.68 & 0.61 & 0.61 & 0.60 & 0.78 \\
\hline $1990 \mathrm{~s}$ & 0.74 & 0.60 & 0.67 & 0.67 & 0.72 \\
\hline $2000 s$ & 0.72 & 0.57 & 0.63 & 0.64 & 0.73 \\
\hline
\end{tabular}

Note: Correlations larger than \pm .30 are significant at $p<.05$. 
Table 7

Individualism: correlations between national culture scores and theoretically relevant indicators.

\begin{tabular}{|c|c|c|c|c|c|}
\hline \multirow[t]{3}{*}{ Indicators } & \multicolumn{5}{|c|}{ Culture scores } \\
\hline & \multirow{2}{*}{$\begin{array}{l}\text { Hofstede's } \\
\text { scores }\end{array}$} & \multicolumn{4}{|c|}{ Meta-analytic scores } \\
\hline & & Overall & $1980 \mathrm{~s}$ & $1990 \mathrm{~s}$ & $2000 s$ \\
\hline $\begin{array}{l}\text { Hofstede's Individualism } \\
\text { scores }\end{array}$ & 1.00 & 0.86 & 0.95 & 0.74 & 0.73 \\
\hline \multicolumn{6}{|l|}{ Human development index } \\
\hline $1970 / 80 \mathrm{~s}$ & 0.64 & 0.66 & 0.68 & 0.63 & 0.63 \\
\hline $1990 \mathrm{~s}$ & 0.63 & 0.63 & 0.64 & 0.63 & 0.63 \\
\hline $2000 s$ & 0.56 & 0.60 & 0.59 & 0.59 & 0.60 \\
\hline \multicolumn{6}{|c|}{ Urban population percentage } \\
\hline $1970 / 80 s$ & 0.53 & 0.52 & 0.55 & 0.51 & 0.53 \\
\hline $1990 \mathrm{~s}$ & 0.47 & 0.46 & 0.47 & 0.48 & 0.48 \\
\hline $2000 s$ & 0.44 & 0.44 & 0.45 & 0.47 & 0.47 \\
\hline \multicolumn{6}{|c|}{ GDP/capita adjusted to PPP } \\
\hline $1970 / 80 s$ & 0.82 & 0.74 & 0.82 & 0.71 & 0.73 \\
\hline $1990 \mathrm{~s}$ & 0.75 & 0.71 & 0.76 & 0.68 & 0.72 \\
\hline $2000 s$ & 0.73 & 0.72 & 0.75 & 0.67 & 0.73 \\
\hline \multicolumn{6}{|l|}{ Divorce rate } \\
\hline $1970 / 80 \mathrm{~s}$ & 0.77 & 0.62 & 0.74 & 0.54 & 0.47 \\
\hline $1990 \mathrm{~s}$ & 0.75 & 0.56 & 0.73 & 0.48 & 0.50 \\
\hline $2000 s$ & 0.59 & 0.53 & 0.55 & 0.50 & 0.47 \\
\hline \multicolumn{6}{|l|}{ Occupants per household } \\
\hline $1970 / 80 s$ & -0.65 & -0.62 & -0.67 & -0.58 & -0.63 \\
\hline $1990 \mathrm{~s}$ & -0.65 & -0.65 & -0.67 & -0.64 & -0.67 \\
\hline $2000 s$ & -0.61 & -0.63 & -0.64 & -0.58 & -0.65 \\
\hline \multicolumn{6}{|c|}{ Number of children residing with parents } \\
\hline $1970 / 80 s$ & -0.59 & -0.52 & -0.62 & -0.46 & -0.50 \\
\hline $1990 \mathrm{~s}$ & -0.54 & -0.55 & -0.58 & -0.50 & -0.55 \\
\hline $2000 \mathrm{~s}$ & -0.48 & -0.54 & -0.54 & -0.51 & -0.55 \\
\hline
\end{tabular}

Table 8

Masculinity: correlations between national culture scores and theoretically relevant indicators.

\begin{tabular}{|c|c|c|c|c|c|}
\hline \multirow[t]{3}{*}{ Indicators } & \multicolumn{5}{|c|}{ Culture scores } \\
\hline & \multirow{2}{*}{$\begin{array}{l}\text { Hofstede's } \\
\text { scores }\end{array}$} & \multicolumn{4}{|c|}{ Meta-analytic scores } \\
\hline & & Overall & $1980 \mathrm{~s}$ & $1990 s$ & $2000 \mathrm{~s}$ \\
\hline $\begin{array}{l}\text { Hofstede's Masculinity } \\
\text { scores }\end{array}$ & 1.00 & 0.85 & 0.85 & 0.75 & 0.56 \\
\hline \multicolumn{6}{|c|}{ Percent women in parliament } \\
\hline $1970 / 80 s$ & -0.49 & -0.43 & -0.54 & -0.41 & -0.48 \\
\hline $1990 \mathrm{~s}$ & -0.44 & -0.43 & -0.48 & -0.45 & -0.51 \\
\hline $2000 s$ & -0.40 & -0.40 & -0.42 & -0.36 & -0.58 \\
\hline \multicolumn{6}{|c|}{ Importance of promotion } \\
\hline $1970 / 80 s$ & 0.63 & 0.43 & 0.64 & 0.32 & 0.34 \\
\hline $1990 s$ & 0.41 & 0.38 & 0.35 & 0.34 & 0.21 \\
\hline $2000 \mathrm{~s}$ & 0.41 & 0.39 & 0.31 & 0.36 & 0.29 \\
\hline \multicolumn{6}{|l|}{ Importance of good pay } \\
\hline $1970 / 80 s$ & 0.46 & 0.44 & 0.42 & 0.31 & 0.36 \\
\hline $1990 s$ & 0.31 & 0.23 & 0.19 & 0.29 & 0.26 \\
\hline $2000 s$ & 0.35 & 0.40 & 0.43 & 0.48 & 0.51 \\
\hline \multicolumn{6}{|c|}{ Patent application per capita, residents } \\
\hline $1970 / 80 s$ & 0.31 & 0.39 & 0.45 & 0.34 & 0.21 \\
\hline $1990 s$ & 0.28 & 0.30 & 0.42 & 0.29 & 0.13 \\
\hline $2000 s$ & 0.28 & 0.29 & 0.43 & 0.31 & 0.14 \\
\hline
\end{tabular}

Note: Correlations larger than \pm .30 are significant at $p<.05$. 
Table 9

Uncertainty Avoidance: correlations between national culture scores and theoretically relevant indicators.

\begin{tabular}{|c|c|c|c|c|c|}
\hline \multirow[t]{3}{*}{ Indicators } & \multicolumn{5}{|c|}{ Culture scores } \\
\hline & \multirow{2}{*}{$\begin{array}{l}\text { Hofstede's } \\
\text { scores }\end{array}$} & \multicolumn{4}{|c|}{ Meta-analytic scores } \\
\hline & & Overall & $1980 \mathrm{~s}$ & $1990 s$ & $2000 \mathrm{~s}$ \\
\hline $\begin{array}{l}\text { Hofstede's Uncertainty } \\
\text { Avoidance scores }\end{array}$ & 1.00 & 0.72 & 0.85 & 0.62 & 0.46 \\
\hline \multicolumn{6}{|l|}{ Inflation } \\
\hline $1970 / 80 s$ & 0.31 & 0.25 & 0.32 & 0.16 & 0.31 \\
\hline $1990 \mathrm{~s}$ & 0.30 & 0.33 & 0.31 & 0.41 & 0.54 \\
\hline $2000 s$ & 0.20 & 0.38 & 0.15 & 0.53 & 0.46 \\
\hline \multicolumn{6}{|c|}{ Corruption perception index } \\
\hline $1970 / 80 s$ & 0.22 & 0.34 & 0.32 & 0.40 & 0.35 \\
\hline $1990 s$ & 0.21 & 0.50 & 0.50 & 0.50 & 0.54 \\
\hline $2000 \mathrm{~s}$ & 0.22 & 0.49 & 0.48 & 0.51 & 0.55 \\
\hline \multicolumn{6}{|c|}{ Crime, assault indices per capita } \\
\hline $1970 / 80 s$ & 0.17 & 0.23 & 0.31 & 0.22 & 0.27 \\
\hline $1990 s$ & 0.24 & 0.37 & 0.30 & 0.42 & 0.43 \\
\hline $2000 \mathrm{~s}$ & 0.27 & 0.39 & 0.36 & 0.39 & 0.47 \\
\hline \multicolumn{6}{|l|}{ Unemployment } \\
\hline $1970 / 80 s$ & 0.17 & 0.22 & 0.25 & 0.20 & 0.52 \\
\hline $1990 s$ & 0.24 & 0.25 & 0.27 & 0.27 & 0.42 \\
\hline $2000 s$ & 0.27 & 0.28 & 0.32 & 0.28 & 0.36 \\
\hline \multicolumn{6}{|c|}{ Patent application per capita, non-residents } \\
\hline $1970 / 80 s$ & -0.19 & -0.50 & -0.41 & -0.50 & -0.43 \\
\hline $1990 \mathrm{~s}$ & -0.24 & -0.37 & -0.38 & -0.40 & -0.61 \\
\hline $2000 s$ & -0.22 & -0.33 & -0.29 & -0.40 & -0.58 \\
\hline \multicolumn{6}{|l|}{ Lending interest rate } \\
\hline $1970 / 80 s$ & 0.25 & 0.24 & 0.34 & 0.08 & 0.27 \\
\hline $1990 \mathrm{~s}$ & 0.36 & 0.39 & 0.42 & 0.54 & 0.45 \\
\hline $2000 \mathrm{~s}$ & 0.23 & 0.28 & 0.25 & 0.39 & 0.48 \\
\hline
\end{tabular}

Fourth, if cultures indeed change over decades, the correlations between meta-analytic indices and external national indicators should be the strongest when they are temporally matched and represent the same decade. In most cases, they are indeed stronger, at times considerably so. For data from the 1980 s, $80 \%$ of correlations were equal or higher if the cultural scores also came from the same time period. For data from 1990 s, $65 \%$ were equal or higher if matched. For data from the 2000s, $85 \%$ were equal or higher. Notably, the theoretically more turbulent Uncertainty Avoidance was substantively improved by using decade-bydecade analyses. Most decade-specific correlations were superior to overall correlations, at times greatly so. For example, the metaanalytic Uncertainty Avoidance index for the 2000s correlates with the corruption perception index for the 2000s at 0.55 , whereas the correlation between the corruption perception index for the 2000s and overall meta-analytic Uncertainty Avoidance score is 0.49 and with Hofstede's Uncertainty Avoidance score from the IBM study only 0.22 .

Further inspection of the results reveals a few additional interesting observations. Most important is that cultural change is not uniform across countries. While overall we see a gradual increase in Individualism and a decrease in Power Distance, Masculinity and Uncertainty Avoidance, the pace and at time the direction of the change varies greatly among the countries. These changes lead to substantial changes in their relative rankings along the cultural dimensions. The biggest shifts towards values of developed capitalist societies have been observed for the countries that experienced changes in their political and economic systems, such as newly independent countries that used to be parts of the former USSR (e.g., Russia, Ukraine) or new booming economies of Latin America (e.g., Brazil, Argentina). At the same time, the changes are hardly noticeable for most "older democracies" such as the U.S. and Western Europe. Figs. 1 and 2 compare cultural change in the U.S. versus Latin America. While cultural values in both regions have been changing over time, the extent of the change is much greater 
and, as is in the case of Power Distance, Latin American countries appear to have become less concerned with power differences compared to the U.S., which has been traditionally scoring lower-than-average on Power Distance.

\section{Discussion}

Reflecting the critical role of culture, cross-cultural research has been on the rise in recent decades. Hofstede's framework has been particularly instrumental in helping quantify culture and integrate the construct of culture in business research. Despite the unmatched popularity of Hofstede's national cultural scores, however, their quality and relevance has often been questioned, particularly with respect to the uni-organizational design, small samples representing some countries, and the age of the data. A number of more recent studies have offered new sets of cultural indices, but due to the high cost of international and longitudinal research, they largely remain subject to the same limitations, namely limited ability to represent their respective populations and containment to a specific time period. Even the largest studies (e.g., House et al., 2004; Schwartz, 1994; Smith et al., 1996) numerous countries were represented by only a few dozen individuals and the data represented a single point in time or a period too short to effectively preclude longitudinal analysis.

The present study is an attempt to address these limitations by offering updated national cultural scores based on a meta-analysis of 451 studies that used models and research designs compatible to that used by Hofstede. Our sample size and representativeness (due to the inclusion of data representing several decades) offers a unique opportunity to explore dynamics of national cultures around the world. The separate sets of cultural scores for different decades may be particularly useful in cases when archival data must be matched with culture indices representing the same time period or when a longitudinal database of national indicators needs to be matched with a similar longitudinal database of cultural indices to explore the relationship between the sets dynamically.

A series of validity checks indicate that our updated cultural scores are more accurate than those offered by Hofstede. An examination of the links between Hofstede's and our metaanalytic indices and a number of theoretically relevant criteria such as demographics, economic and societal indicators confirmed that the precision of Hofstede's scores has been decreasing over time. While Hofstede's scores correlated remarkably strongly with theoretically relevant indicators from the 1980s, the correlations typically weakened for each subsequent decade thereafter. At the present rate of validity degradation, Hofstede's scores will no longer recognizably represent world culture within a few decades. At the same time, the meta-analytic scores normally have higher correlations with theoretically relevant indicators than Hofstede's scores do. The differences are especially noticeable when meta-analytic scores and external criteria represent matching decades. In addition to relying on a larger and more representative and generalizable sample, the present study also provided cultural indices separately for each of the several past decades. The meta-analytic scores were derived from hundreds of smaller studies that used the same methodology to survey the same populations (nations) at different times, thereby providing a longitudinal dataset.

\subsection{Managerial relevance}


Hofstede's cultural scores have been successfully used to explain and predict a wide range of business and economics phenomena and processes in human resource management (Ramamoorthy \& Carroll, 1998), international trade and cooperation (Kogut \& Singh, 1988), marketing (Yeniyurt \& Townsend, 2003), accounting and audit (Yamamura, Frakes, Sanders, \& Ahn, 1996), entrepreneurship (Mueller \& Thomas, 2001) and many more. As a result, Hofstede's work provided a foundation for a wide range of corporate cross-cultural training programs. It has become an integral part of international business and management curricula (Bhawuk \& Brislin, 2000; Landis, Bennett, \& Bennett, 2006). The indices proved especially useful in pre-departure training for expatriates and multi-cultural workgroup development programs (Littrell, Salas, Hess, Paley, \& Riedel, 2006).

While Hofstede's original national cultural indices are likely to remain relevant for some time, the updated sets of meta-analytic cultural scores offered in the present study may offer a more current view on the cultural map of the world. The longitudinal nature of the dataset presented here could also help enhance cross-cultural business and executive education programs by providing a foundation for discussions on cultural change, acculturation, cultural differences within countries, and other important issues.

Furthermore, the meta-analytic cultural indices allow for new research directions into the interplay of culture and various national indicators. For example, the relationship between culture and various phenomena no longer need to be done by correlating older Hofstede's indices with more recent measures. Indicators or phenomena relevant to culture can now be matched with cultural indices from the corresponding decade, largely eliminating the temporal mismatch between measures. Similarly, the longitudinal cultural dataset could be used in conjunction with other longitudinal datasets, such as those compiled by the World Bank, Transparency International, United Nations or other similar organizations, to address a wide range of research questions that had been previously out of reach due to restrictions imposed by availability of only cross-sectional culture datasets. In particular, the entire research area of what causes culture (i.e., culture as a consequence), which is almost completely unexplored (Steel \& Taras, 2010), becomes ripe for consideration with a longitudinal cultural dataset. The new findings would provide better guidance for practitioner managers seeking to maximize performance of their organizational in cross-cultural context.

\subsection{Limitations and directions for future research}

Albeit more accurate and representative than Hofstede's indices from the 1960s and 1970s, the meta-analytic indices are certainly not perfect. A few cultural scores in our report appear to deviate too much from their expected positions and a number of validity indicators fell short of supporting the predicted trends. The problems are most likely due to two issues. First, perfect commensurability among cultural measures cannot be achieved, and this injects method variance into our scores. However, this is an issue for any meta-analytic endeavor (Steel, Schmidt, \& Shultz, 2008). Second, the level of sampling error is dependent on the participant choices of the original researchers who collected the source data. Steel and Taras (2010) have found, for example, that cultural scores are influenced by age, gender, education and socioeconomic status ofthe respondent. In the same way that Hofstede's IBM sample may have affected his scores, random variations in researchers' sampling strategies could have affected ours, though arguably to far a lesser degree. 
It should also be noted that in our attempt to replicate Hofstede's original approach as closely as possible, we share many of the limitations of Hofstede's original work. For example, we relied on Hofstede's dimensions despite concerns that they may not adequately capture all aspects of culture. Unfortunately, although alternative and potentially better models of culture have been developed (e.g., House et al., 2004; Schwartz, 1994; Smith et al., 1996), they have not been popular enough to generate sufficient data for meta-analysis.

Although our dataset was quite large, the number of samples for some smaller countries was still low. Of course, given that some of the national averages used by Hofstede were based on responses from less than a hundred individuals, even a single additional sample could substantially improve the estimates. Yet, any meta-analytic indices based on a few data points should be used with caution. Consequently, our tables provide details about the number of samples and the number of respondents representing each score. This permits statistical analyses that take into account the sampling error associated with any score, such as Weighted Least Square (WLS) multiple regression (Steel \& Kammeyer Mueller, 2002).

A number of interesting and important research questions were beyond the scope of present study and may be addressed in the future. For example, we largely ignored the issue of cultural variability within countries and instead focused on national means as has traditionally been done in all earlier large-scale culture comparison studies. Yet, limitations of the meanbased approach are particularly salient in cross-cultural research (Taras et al., 2009). A metaanalytic study into the antecedents and consequences of cultural variability within countries could be very fruitful.

Although the results of the present study suggest that cultures do change, the focus of the present study was on the meta-analytic indices and how they were derived, not to provide an analysis regarding trends and nature of the observed changes. However, a closer examination into the nature and pattern of this change is warranted in future studies. In particular, this dataset can offer some insight into Modernization Theory, which postulates that "modernization" of economic, political, and social sectors brings about a change in cultural values that reflect new conditions (Bell, 1973; Kerr, Dunlop, Harbison, \& Myers, 1960). Likewise, the meta-analytic data reported here may be used to test cultural Convergence theory that postulates that globalization and international interactions aid cultural exchange and would eventually lead to an emergence of a universal global culture (Webber, 1969).

Finally, future researchers may want to reexamine earlier findings regarding culture's role and effects. Hofstede's indices have been used to assess the relationships between cultural values and hundreds of different organizational and societal phenomena. Due to the limitations of Hofstede's scores discussed earlier, it is likely that when his scores were used, in particular in more recent studies, the findings underestimated true effects. Our meta-analytic cultural scores present an opportunity to revisit those research questions with more precise tools.

\section{Conclusion}

The popularity of Hofstede's cultural scores clearly demonstrates the need for national cultural rankings. However, the relevance of Hofstede's scores has been gradually eroding with each passing decade. We revitalize this extremely useful database by putting forth a new set of national cultural rankings, one that we argue is more accurate than that offered by Hofstede or other cross-cultural comparison studies. Our dataset is based on metaanalytic aggregation of hundreds of earlier studies. This approach yields a dataset that is larger and more representative 
than any individual study can offer. Furthermore, the meta-analytic approach allows for addressing cultural change by offering separate sets of indices for different time periods. This strategy is not easily duplicated through other methodologies. For example, a dedicated research team would require unprecedented resources and must retain focus over a period of time that is longer than most people's active professional careers. Furthermore, this team cannot collect data before they start; that is, go back in time when such a database has not yet been suggested. Metaanalytic aggregation can do this and can continue on indefinitely, with updates occurring as long as people gather primary data.

Given these advantages, we argue that this meta-analytic methodology should be continued. With no sign that Hofstede's model is waning in popularity, we can expect future iterations of this database to continue every decade for several decades to come. We also recommend that this strategy should be replicated across any other national self-reportindicators with sufficient history and depth of use, with subjective well-being coming quickly to mind (Veenhoven, 2010). If adopted, the study of culture is about to become even more dynamic and exciting than it already is. We will start considering how and why cultures change over time and delays between actions and causes. This will enable us to predict cultural change as well as purposely influence culture itself.

\section{References}

Adams, M. (2005). American backlash: The untold story of social change in the United States. New York: The Penguin Group.

Bell, D. (1973). The coming of post-industrial society. New York, NY: Basic Books.

Bhawuk, D. P. S., \& Brislin, R. W. (2000). Cross-cultural training: A review. Applied Psychology: An International Review, 49(1): 162-191.

Chakrabarty, S. (2009). The influence of national culture and institutional voids on family ownership of large firms: A country level empirical study. Journal of International Management, 15(1): 32-45.

Dorfman, P., \& Howell, J. P. (1988). Dimensions of national culture and effective leadership patterns: Hofstede revisited. In R. N. Farmer \& E. G. McGoun (Eds.), Advances in international comparative management (pp. 127-150). London, UK: JAI Press.

FH. (2010). Freedom House: Freedom in the World Data Series. http://www.freedomhouse. org/template.cfm?page $=15$.

Furrer, O., Liu, B., \& Sudharshan, D. (2000). The relationships between culture and service quality perceptions: Basis for cross-cultural market segmentation and resource allocation. Journal of Service Research, 2(4): 355-371.

HF. (2010). Heritage Foundation reports. http://www.heritage.org/Research. Hofstede, G. (1980). Culture's consequences: International differences in work-related values. Beverly Hills, CA: Sage Publications.

Hofstede, G. (2001). Culture's consequences: Comparing values, behaviors, institutions and organizations across nations (2nd ed.). London: Sage Publications.

Hofstede, G. (2002). Dimensions do not exist: A reply to Brendan McSweeney. Human Relations, 55(11): 1355-1361.

House, R. J., Hanges, P. J., Javidan, M., Dorfman, P. W., \& Gupta, V. (Eds.). (2004). Culture, leadership, and organizations: The GLOBE study of 62 societies. Thousand Oaks, CA: Sage Publications. 
Hunter, J. E., \& Schmidt, F. L. (2004). Methods of meta-analysis: Correcting error and bias in research findings (2nd ed.). Newbury Park, CA: Sage Publications.

Inglehart, R., \& Baker, W. E. (2000). Modernization, cultural change, and the persistence of traditional values. American Sociological Review, 65(1): 19-51.

Kerr, C., Dunlop, J. T., Harbison, F. H., \& Myers, C. A.(1960). Industrialism and industrial man: The problems of labor and management in economic growth. London: Heinemann.

Kogut, B., \& Singh, H. (1988). The effect of national culture on the choice of entry mode. Journal of International Business Studies, 19(3): 411-432.

Kroeber, A. L., \& Kluckhohn, C. (1952). Culture: A critical review of concepts and definitions. New York: Vintage Books.

Kuhn, M. H., \& McPartland, R. (1954). An empirical investigation of self attitudes. American Sociological Review, 19: 68-76.

Landis, D., Bennett, J., \& Bennett, M. (Eds.). (2006). Handbook of intercultural training (3rd ed.). Thousand Oaks, CA: Sage Publications.

Littrell, L. N., Salas, E., Hess, K. P., Paley, M., \& Riedel, S. (2006). Expatriate preparation: A critical analysis of 25 years of cross-cultural training research. Human Resource Development Review, 5(3): 355-388.

Matsumoto, D. (1999). Culture and self: An empirical assessment of Markus and Kitayama's theory of independent and interdependent self-construal. Asian Journal of Social Psychology, 2(3): 289-310.

Maznevski, M. L., DiStefano, J. J., Gomez, C., Noorderhaven, N. G., \& Wu, P. C. (2002). Cultural dimensions at the individual level of analysis: The cultural orientation framework. International Journal of Cross-Cultural Management, 2(3): 275-295.

McSweeney, B. (2002). Hofstede's model of national cultural differences and their consequences: A triumph of faith—A failure of analysis. Human Relations, 55(1): 89118.

Mueller, S. L., \& Thomas, A. S. (2001). Culture and entrepreneurial potential: A nine country study of locus of control and innovativeness. Journal of Business Venturing, 16(1): 5169.

Murphy, E., Gordon, J., \& Mullen, A. (2004). A preliminary study exploring the value changes taking place in the US since the September 11, 2001 terrorist attack on the World Trade Center in New York. Journal of Business Ethics, 50(1): 81-96.

Newburry, W., \& Yakova, N. (2006). Standardization preferences: A function of national culture work interdependence and local embeddedness. Journal of International Business Studies, 37(1): 44-60.

Ng, T. W. H., Sorensen, K. L., \& Yim, F. H. (2009). Does the job satisfaction-job performance relationship vary across cultures? Journal of Cross-Cultural Psychology, 40(5): 1-36.

Olivas-Luja'n, M. R., Harzing, A. W., \& McCoy, S. (2004). September 11, 2001: Two quasiexperiments on the influence of threats on cultural values and cosmopolitanism. International Journal of Cross Cultural Management, 4(2): 211-228.

Oyserman, D., Coon, H. M., \& Kemmelmeier, M. (2002). Rethinking individualism and collectivism: Evaluation of theoretical assumptions and meta-analyses. Psychological Bulletin, 128(1): 3-72.

Ralston, D. A., Holt, D. H., Terpstra, R. H., \& Kai Cheng, Y. (1997). The impact of national culture and economic ideology on managerial work values: A study of the United States, Russia, Japan and China. Journal of International Business Studies, 28(1): 177-207. 
Ramamoorthy, N., \& Carroll, S. J. (1998). Individualism/collectivism orientations and reactions toward alternative human resource management practices. Human Relations, 51(5): 571588.

Rokeach, M. (1973). The nature of human values. New York: Free Press.

Schimmack, U., Oishi, S., \& Diener, E. (2005). Individualism: A valid and important dimension of cultural differences between nations. Personality and Social Psychology Review, 9(1): $17-31$.

Schwartz, S. H. (1994). Beyond individualism/collectivism: New cultural dimensions of values. In U. Kim, H. C. Triandis, C. Kagitcibasi, S. C. Choi, \& G. Yoon (Eds.), Individualism and collectivism: Theory, methods and applications (pp. 85-119). London: Sage Publications.

Shah, G. (2009). The impact of economic globalization on work and family collectivism in India. Journal of Indian Business Research, 1.(2): 95-118.

Sharpe, D. (1997). Of apples and oranges, file drawers and garbage: Why validity issues in metaanalysis will not go away. Clinical Psychology Review, 17(8): 881- 901.

Singelis, T. M., Triandis, H. C., Bhawuk, D. P. S., \& Gelfand, M. J. (1995). Horizontal and vertical dimensions of individualism and collectivism: A theoretical and measurement refinement. Cross-Cultural Research, 29(3): 240-275.

Smith, P. B. (2000). Changes in the generation gap 1972-1998: GSS Social Change Report (Vol. 43). Chicago, IL: National Opinion Research Center, University of Chicago.

Smith, P. B., Dugan, S., \& Trompenaars, F. (1996). National culture and the values of organizational employees: A dimensional analysis across 43 nations. Journal of CrossCultural Psychology, 27(2): 231-264.

Steel, P., \& Kammeyer-Mueller, J. D. (2002). Comparing meta-analytic moderator estimation techniques under realistic conditions. Journal of Applied Psychology, 87(1): 96-111.

Steel, P., Schmidt, J., \& Shultz, J. (2008). Refining the relationship between personality and subjective well-being. Psychological Bulletin, 134(1): 138-161.

Steel, P., \& Taras, V. (2010). Culture as a consequence: A multilevel multivariate metaanalysis of the effects of individual and country characteristics on work-related cultural values. Journal of International Management, 16(3): 211-233.

Taras, V. (2009). Catalogue of instruments for measuring culture. Retrieved January 1, 2009, from http://vtaras.com/files/Culture_Survey_Catalogue.pdf.

Taras, V., Kirkman, B. L., \& Steel, P. (2010). Examining the impact of culture's consequences: A three-decade, multi-level, meta-analytic review of Hofstede's cultural value dimensions. Journal of Applied Psychology, 95(3): 405-439.

Taras, V., Rowney, J., \& Steel, P. (2009). Half a century of measuring culture: Approaches, challenges, limitations and suggestions based on the analysis of 112 instruments for quantifying culture. Journal of International Management, 15(4): 357-373.

Taras, V., Sarala, R., \& Muchinsky, P. (2010b). Opposite ends of the same stick: Multimethod test of independence of individualism and collectivism. Paper presented at the Academy of International Business, Rio de Janeiro, Brazil.

Taras, V., \& Steel, P. (2009). Beyond Hofstede: Challenging the ten testaments of cross-cultural research. In C. Nakata (Ed.), Beyond Hofstede: Culture frameworks for global marketing and management (pp. 40-61). Chicago, IL: Macmillan/Palgrave. 
Taras, V., Steel, P., \& Kirkman, B. L. (2010). Negative practice-value correlations in the GLOBE data: Unexpected findings, questionnaire limitations and research directions. Journal of International Business Studies, 41(8): 1330-1338.

Triandis, H. C., \& Gelfand, M. J. (1998). Converging measurement of horizontal and vertical individualism and collectivism. Journal of Personality and Social Psychology, 74(1): $118-128$.

Triandis, H. C., Leung, K., Villareal, M. J., \& Clack, F. L. (1985). Allocentric versus idiocentric tendencies: Convergent and discriminant validation. Journal of Research in Personality, 19: 395-415.

UNO. (2010). UN data: A world of information (Publication no. http://data.un.org/).

Veenhoven, R. (2010). World database of happiness. Retrieved March 1, 2011, from http://worlddatabaseofhappiness.eur.nl/hap_quer/hqi_fp.htm.

Vitell, S. J., Paolillo, J. G. P., \& Thomas, J. L. (2003). The perceived role of ethics and social responsibility: A study of marketing professionals. Business Ethics Quarterly, 13(1): 6386.

Voronov, M., \& Singer, J. A. (2002). The myth of individualism-collectivism: A critical review. Journal of Social Psychology, 142(4): 461-480.

Wagner, J. A., \& Moch, M. K. (1986). Individualism-collectivism: Concept and measure. Group \& Organization Studies, 11(3): 280-303.

WB. (2010). World bank data [on-line database]. http://data.worldbank.org/.

Webber, R. A. (1969). Convergence or divergence? Columbia Journal of World Business, 4(3): $75-84$.

Yamamura, J. H., Frakes, A. H., Sanders, D. L., \& Ahn, S. K. (1996). A comparison of Japanese and U.S. auditor decision-making behavior. International Journal of Accounting, 31(3): 347-363.

Yeniyurt, S., \& Townsend, J. D. (2003). Does culture explain acceptance of new products in a country? An empirical investigation. International Marketing Review, 20(4): 377-395. 